\title{
Unveiling the biochemistry of the epigenetic regulator SMYD3
}

\section{Supplementary information}

Edoardo Fabini, ${ }^{\dagger},, \#$ Vladimir O. Talibov, ${ }^{\boldsymbol{q}, \#}$ Filip Mihalic, 9 Marina Naldi, ${ }^{\dagger, \S}$ Manuela Bartolini, ${ }^{\dagger}$ Carlo Bertucci, ${ }^{\dagger}$ Alberto Del Rio, ${ }^{\ddagger}, \|$ and U. Helena Danielson*,,$\perp$

$\dagger$ Department of Pharmacy and Biotechnology, Alma Mater Studiorum University of Bologna, Bologna, Italy

$\ddagger$ Institute of Organic Synthesis and Photoreactivity (ISOF), National Research Council (CNR), Bologna, Italy

9Department of Chemistry - BMC, Uppsala University, Uppsala, Sweden $\S$ Center for Applied Biomedical Research (C.R.B.A.), S. Orsola-Malpighi Hospital, Bologna, Italy

|| Innovamol consulting Srl, Modena, Italy

$\perp$ Science for Life Laboratory, Uppsala University, Uppsala, Sweden \#Contributed equally to this work

E-mail: helena.danielson@kemi.uu.se 


\section{SMYD3 crystallization, data collection and analysis}

For the initial screening, the SMYD3 concentration was kept constant at $9 \mathrm{mg} \mathrm{mL} \mathrm{m}^{-1}$. Further trials were conducted with concentration varying from 6 to $12 \mathrm{mg} \mathrm{mL}^{-1}$. The protein concentration was adjusted with $50 \mathrm{mM}$ Tris, $150 \mathrm{mM} \mathrm{NaCl}, p \mathrm{H}$ 8.0.

Initial crystallisation hits were obtained using Morpheus II crystallisation screen (Molecular Dimensions, UK) in a sitting drop manner, with a total drop volume of $0.4 \mu \mathrm{L}$ and using Mosquito liquid handling system (TTP Labtech Ltd., UK). No crystalline material was observed for the N-terminally his-tagged form of the protein, hits with the untagged form were elaborated in a hanging drop vapour diffusion set up using a 1:1 protein-to-reservoir ratio, $2 \mu \mathrm{L}$ total drop volume, at $20^{\circ} \mathrm{C}$.

Two different forms of SMYD3 crystals were obtained. On average, crystals nucleated within one day and grew to their maximal dimensions within one week. Form I (3dimensional pinacoid-like crystals) appeared in $100 \mathrm{mM}$ Tris $p \mathrm{H} 8.0-8.5,8-12 \%$ PEG8k, $50 \mathrm{mM}$ magnesium acetate. Both form I and form II (columnar crystals or needle clusters) nucleated in $100 \mathrm{mM}$ Tris $p \mathrm{H}$ 7.75-8.25, $100 \mathrm{mM}$ magnesium acetate, 10-14\% PEG3350. Upon supplementation of the reservoir solution with DMSO to 10-20\% (v/v), the latter conditions promoted exclusively the nucleation and growth of form II crystals. Addition of zinc salts or thiol di-exchange reagents to the reservoir solutions inhibited the nucleation.

Crystals were cryoprotected in a reservoir solution supplemented with $10 \%$ glycerol (DMSO-containing reservoir solutions) or $20 \%$ 1,5-pentanediol (DMSO-free solutions) and flash-frozen in a liquid nitrogen. Diffraction data was collected at the European Synchrotron Radiation Facility (ESRF, Grennoble, France) beamline ID24. Indexing and integration was performed on site using FAST DP, ${ }^{1,2}$ scaling was done with AIMLESS. ${ }^{3}$ The protein struc-

ture was solved using PhaserMR, ${ }^{4}$ utilising SMYD3 structure 5EX0 as a search model. ${ }^{5}$ Data collection parameters are given in Table S1. 


\section{Figures}

(A)

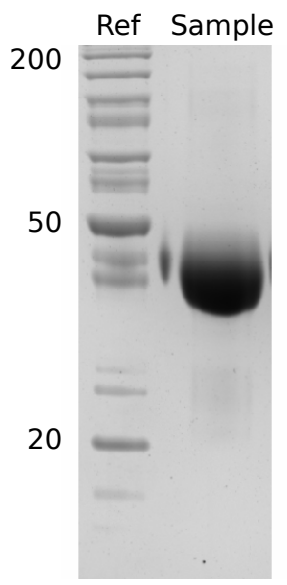

(B)

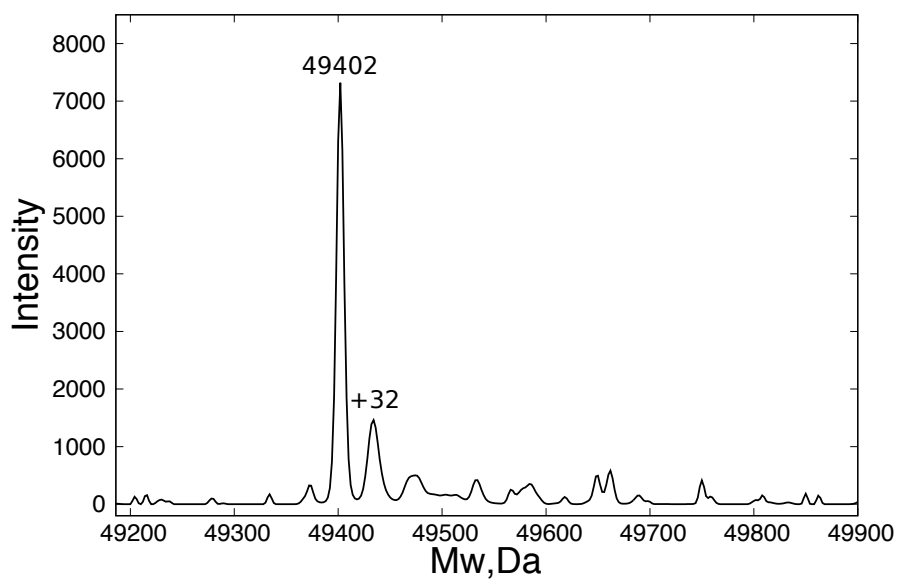

Figure S1: SMYD3 purity analysis. Panel A - 12.5\% SDS PAGE gel, stained with Coomassie G250; protein load - $15 \mu \mathrm{g}$. Panel B - deconvoluted ESI-MS spectra of SMYD3, obtained under denaturing conditions. Calculated molecular weight -49392 , found -49402 . 
Peptide 6: VEGFR ${ }_{824-845}$
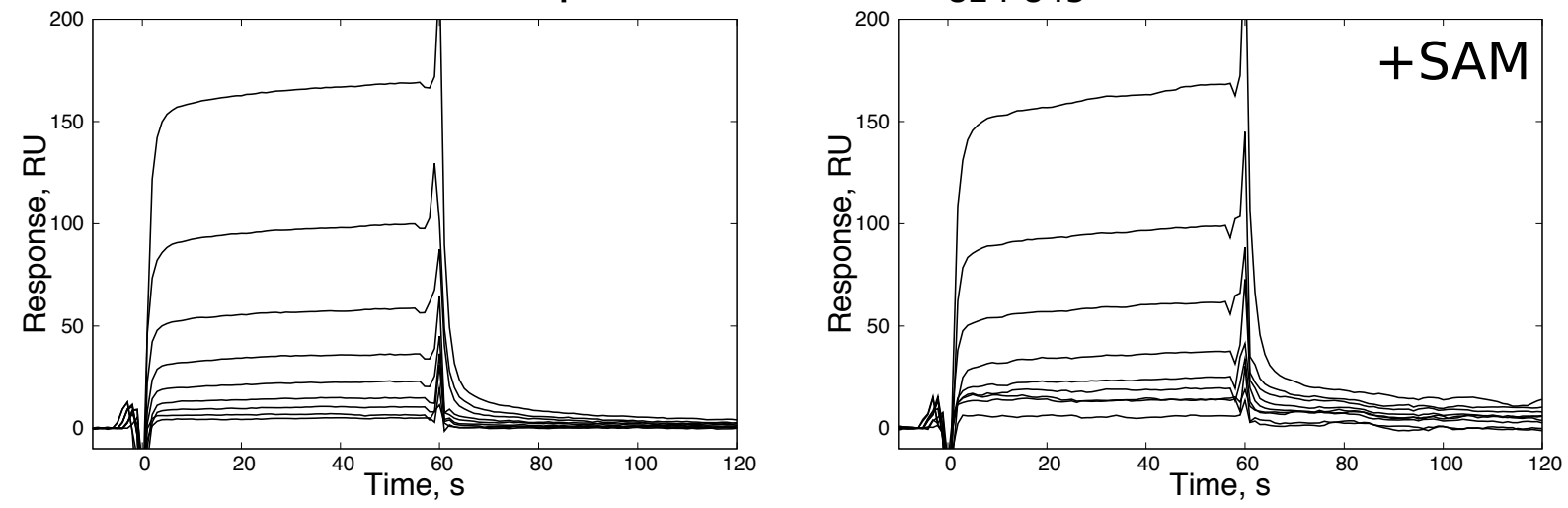

Peptide 8: $\mathrm{H}_{3 K} 4_{1-26}$
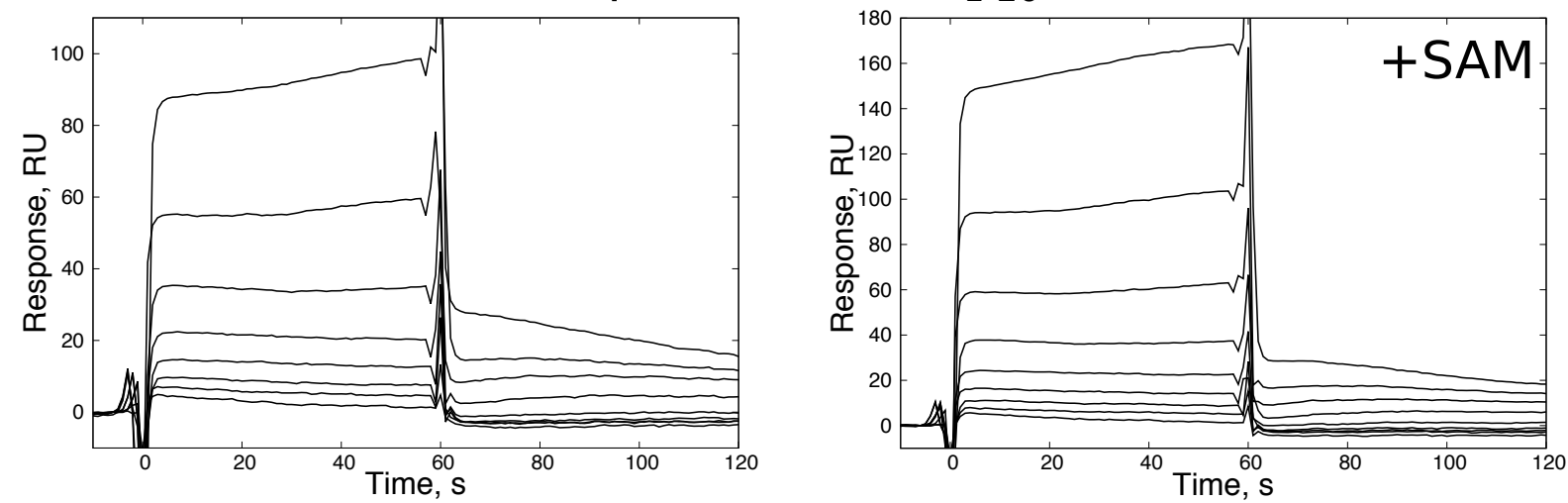

Peptide 9: $\mathrm{H}_{4 \mathrm{~K} 5}{ }_{1-26}$
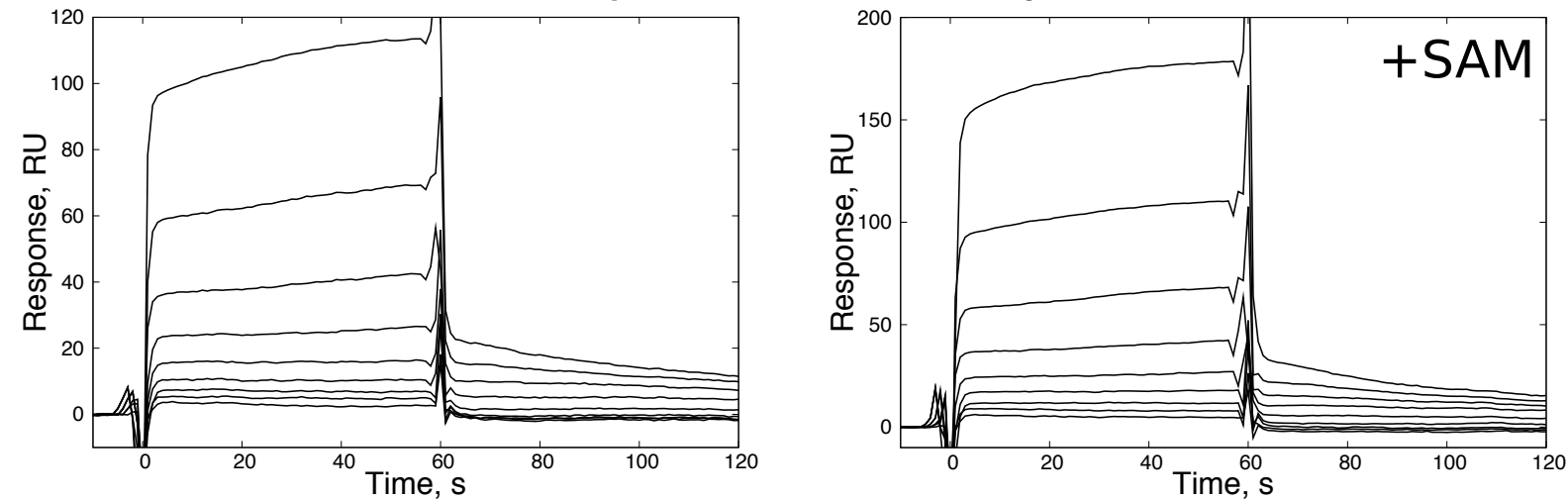

Figure S2: Analysis of interactions between SMYD3 and peptides $\mathbf{6 , 8}$ and $\mathbf{9}$. Peptides were prepared in concentration series starting from $100 \mu \mathrm{M}$. The SMYD3-peptide interactions were weak and had a complex profile. 
(A)

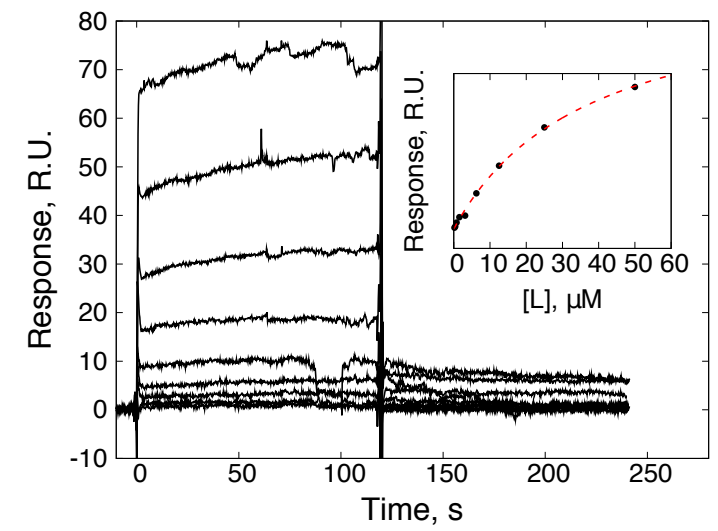

(B)

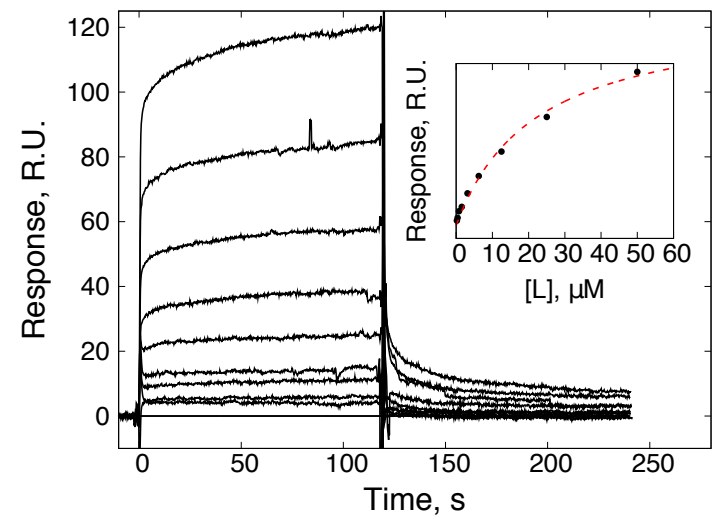

Figure S3: Reversed interaction kinetic analysis between SMYD3 and MAP3K2-based peptide. The biotinylated peptide was immobilized on a streptavidin-functionalized sensor surface, the protein was injected at various concentrations in absence $(\mathbf{A})$ and presence $(\mathbf{B})$ of SAM. Insets correspond to Langmuir isotherms, derived from the signal levels at the end of the protein injections.

(A)

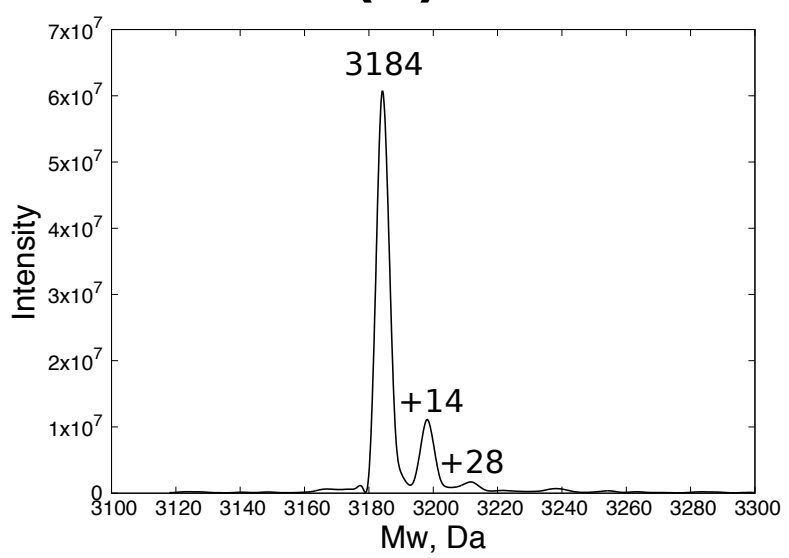

(B)

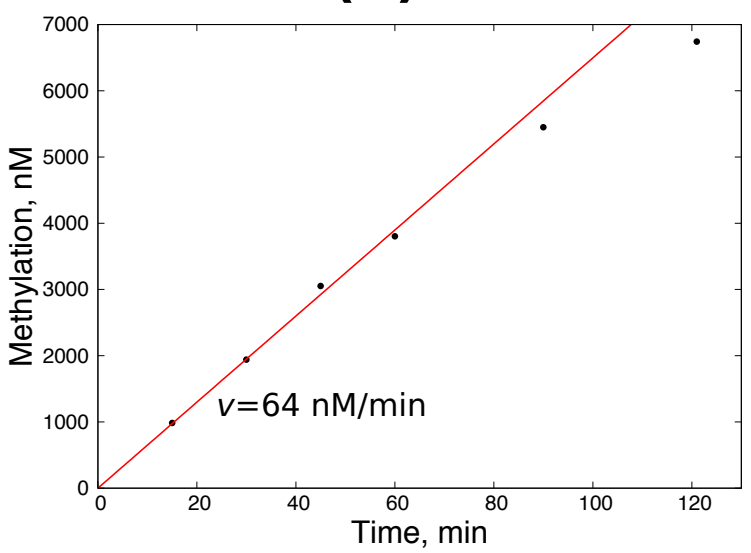

Figure S4: ESI-MS-based SMYD3 activity assay. Peptide 1 ( $M w 3180$ Da) was used as a substrate. Panel A - deconvoluted mass spectra of the methylated peptide; product was quantified as the sum of all methylated forms. Panel $\mathbf{B}$ - reaction progress curve for the following conditions: $0.6 \mu \mathrm{M}$ SMYD3, $50 \mu \mathrm{M}$ SAM, $25 \mu \mathrm{M}$ peptide $\mathbf{1}$. The observable rate of methylation was linear for the first $60 \mathrm{~min}$. 


\section{Tables}

Table S1: Summary of diffraction data. Parameters for a higher resolution shell are given in parenthesis.

\begin{tabular}{l|ll} 
& \multicolumn{1}{|c}{ Form I } & \multicolumn{1}{c}{ Form II } \\
\hline Beamline & \multicolumn{2}{|c}{$\begin{array}{c}\text { ESR ID24 } \\
\text { Wavelength }\end{array}$} \\
Space group & $P_{2_{1}}$ & $P_{2_{1} 2_{1} 2_{1}}$ \\
Unit cell parameters, $\AA, \circ$ & $a=52.5, b=117.5$, & $a=60.8, b=66.1$, \\
& $c=86.1 ; \beta=92.6$ & $c=107.8$ \\
Resolution limits, $\AA$ & $47.9-1.78(1.81-1.78)$ & $56.3-1.6(1.63-1.6)$ \\
N. of reflections & $502016(23625)$ & $329524(15591)$ \\
N. of unique reflections & $98226(4838)$ & $58044(2905)$ \\
$R_{\text {merge }}, \%$ & $6.1(170.3)$ & $8.7(84)$ \\
mean $I / \sigma(I)$ & $10.3(0.8)$ & $8.9(1.7)$ \\
$C C_{1 / 2}$ & $1(0.51)$ & $1(0.67)$ \\
Completeness, $\%$ & $98.8(97.7)$ & $99.9(99.9)$ \\
Multiplicity & $5.1(4.9)$ & $5.7(5.4)$ \\
\hline Chains per asym. unit & 2 & 1
\end{tabular}

Table S2: SMYD3 melting temperature $\left(T_{m}\right)$ as a function of the buffer $p H$. Melting curves were acquired using intrinsic tryptophan fluorescence; ni - no inflection point was observed. The buffer systems composed of $100 \mathrm{mM}$ buffering agent and $200 \mathrm{mM} \mathrm{NaCl}$.

\begin{tabular}{lll|l}
$p \mathrm{H}$ & DMSO, $\%$ & \multicolumn{1}{c}{ Buffer } & $T_{m},{ }^{\circ} \mathrm{C}$ \\
\hline 5.0 & 0 & Citrate & ni \\
5.5 & 0 & Citrate & ni \\
6.0 & 0 & MES & ni \\
6.5 & 0 & MES & 45 \\
7.0 & 0 & bis-Tris & 49 \\
7.5 & 0 & HEPES & 51 \\
8.0 & 0 & Tris & 51 \\
8.0 & 2 & Tris & 50 \\
8.0 & 4 & Tris & 50 \\
8.0 & 6 & Tris & 49 \\
8.0 & 8 & Tris & 48 \\
8.0 & 10 & Tris & 48 \\
8.5 & 0 & Bicine & 49 \\
9.0 & 0 & Bicine & 49 \\
9.5 & 0 & Ethanolamine & ni
\end{tabular}




\section{References}

(1) Kabsch, W. Integration, scaling, space-group assignment and post-refinement. Acta Crystallographica Section D: Biological Crystallography 2010, 66, 133-144.

(2) Winter, G.; McAuley, K. E. Automated data collection for macromolecular crystallography. Methods 2011, 55, 81-93.

(3) Winn, M. D.; Ballard, C. C.; Cowtan, K. D.; Dodson, E. J.; Emsley, P.; Evans, P. R.;

Keegan, R. M.; Krissinel, E. B.; Leslie, A. G.; McCoy, A. Overview of the CCP4 suite and current developments. Acta Crystallographica Section D: Biological Crystallography 2011, 67, 235-242.

(4) McCoy, A. J.; Grosse-Kunstleve, R. W.; Adams, P. D.; Winn, M. D.; Storoni, L. C.; Read, R. J. Phaser crystallographic software. Journal of Applied Crystallography 2007, 40, 658-674.

(5) Fu, W.; Liu, N.; Qiao, Q.; Wang, M.; Min, J.; Zhu, B.; Xu, R.-M.; Yang, N. Structural basis for substrate preference of SMYD3, a SET domain-containing protein lysine methyltransferase. Journal of Biological Chemistry 2016, 291, 9173-9180. 